\title{
Decay Characteristics of Neutron Excess Sodium Nuclei
}

Joseph Bevelacqua

Funding: The author(s) received no specific funding for this work.

Potential competing interests: The author(s) declared that no potential competing interests exist.

\section{Abstract}

In neutron star mergers, neutron excess nuclei and the r-process are important factors governing the production of heavier nuclear systems. An evaluation of sodium nuclei suggests that the heaviest $Z=11$ nucleus will have mass 45 with filling of the $2 p_{1 / 2}$ neutron shell. $A=36-45$ sodium isotopes have limited experimental half-life data, but the model predicts beta decay half-lives in the range of 0.551 $1.31 \mathrm{~ms}$. Based on previous calculations for $Z=9,10,20,26$, and 30 systems, these results likely overestimate the half-lives of $A=36-45$ neutron excess sodium nuclei.

\subsection{Introduction}

The nucleosynthesis of heavy elements occurs by three basic processes that add protons or neutrons to a nuclear system ${ }^{1,2}$. The $p$-process adds protons and the $s$ - or slow process and $r$ - or rapid process adds neutrons. Capture of protons by nuclear systems produces predominantly proton-rich nuclei that tend to decay by positron emission and electron capture ${ }^{1,2}$. Neutron capture creates neutron-rich nuclei, and the resulting nuclear systems depend upon the rate of neutron addition and the beta decay rates of the residual nuclei.

In the s-process neutron capture chain, the time between successive neutron captures is sufficiently long for the product nucleus to beta decay to a stable system. Within the r-process, the time between neutron captures is too short to permit decays except for very rapid beta transitions. Therefore, the rprocess must occur in an environment that has a high density of neutrons. The s-process typically occurs in red giant stars. The r-process occurs in a variety of astronomical events, including supernovae explosions and stellar mergers.

Binary neutron star or neutron star and stellar-mass black hole mergers can form a massive rotating torus around a spinning black hole ${ }^{1}$. The matter ejected from these structures and from supernovae explosions is an important source of rapid neutron capture (r-process) nucleosynthesis ${ }^{1}$. Fully understanding the r-process requires knowledge of the properties of neutron excess nuclei involved in creating heavy nuclear systems. Unfortunately, the majority of these neutron excess systems have never been studied ${ }^{2}$. 
Closing this knowledge gap was a motivation for funding facilities for rare-isotope beams (FRIB) constructed at research facilities located around the world. These facilities are located at RIKEN (Japan) ${ }^{3,4}$, GSI (Germany) $)^{5}$, and Michigan State University (US) ${ }^{6}$. The FRIB facilities enable a new class of experiments to determine the physical properties needed by theoretical models of the structure of unstable neutron excess nuclei. Theoretical studies would complement the forthcoming experiments that will provide critical information on the unstable nuclei that must be understood in order to explain nuclear abundances observed in the universe ${ }^{2}$. In particular, the study of neutron excess systems and their decay properties are significant considerations in understanding the r-process, and its importance in producing the observed elements in the universe.

The study of neutron excess systems is also important for studying nuclear decay properties, nuclear structure under extreme conditions, and nuclear reaction mechanisms. Existing theoretical models have not been extensively applied to many of these neutron excess nuclei.

This paper attempts to partially fill the void by calculating the decay properties of neutron excess systems that are important in nucleosynthesis. These theoretical studies should also assist in planning future experiments associated with neutron excess systems that are far removed from the line of stability.

Neutron excess nuclei that merit study occur throughout the Periodic Table $e^{2-7}$ including nuclei in the $Z$ $\leq 32$ range $^{7}$. Although neutron excess nuclei occur throughout the Periodic Table, this paper focuses on sodium systems as part of a continuing investigation of neutron excess nuclei that are of potential astrophysical significance ${ }^{8-12}$. Previous publications addressed neutron excess calcium ${ }^{8}$, iron ${ }^{9}$, fluorine ${ }^{10}$, zinc $^{11}$, and neon ${ }^{12}$ systems.

The study of light nuclear systems, including sodium, is important for a comprehensive astrophysical interpretation of nucleosynthesis. For example, Terasawa et al. ${ }^{13}$ studied the role of light neutron-rich nuclei during r-process nucleosynthesis in supernovae.

In the neighboring fluorine systems, Recio-Blanco et al. ${ }^{14}$ noted the importance of these nuclei in nucleosynthesis, but observed that knowledge of excess neutron $Z=9$ systems and their associated properties are not well established. Mowlavi et al. ${ }^{15}$ also investigated the nucleosynthesis of fluorine with a focus on asymptotic giant branch stars. Ref. 1 noted that most previous studies of the r-process have concentrated on the synthesis of heavy unstable nuclei. However, in extreme environments such as those encountered in a supernova, light-mass nuclei are also expected to provide an important role in the production of r-process elements. Specifically, Ref. 13 noted that light neutron excess systems can significantly affect the heavy-element abundances.

A recent study of fluorine isotopes in intermediate-mass stellar systems ${ }^{16.17}$ concluded that oxygen fusion could occur at lower densities than initially assumed. This result suggests that intermediate-mass stars are more likely to encounter thermonuclear excursion rather than undergoing gravitational collapse. The resulting white dwarf stars would predominantly contain oxygen, neon, and magnesium. This result was a direct consequence of the nuclear structure of ${ }^{20} \mathrm{~F}$, and its influence on the beta decay to the ${ }^{20} \mathrm{Ne}$ system $^{16,17}$. Refs. 16 and 17 further support the study of neutron excess sodium systems in understanding 
the nucleosynthesis of heavier elements.

Additional neutron excess systems in sodium and neighboring nuclei were conducted by fragmentation of $345 \mathrm{MeV} /$ nucleon ${ }^{48} \mathrm{Ca}$ ions at the RIKEN Radioactive Isotope Beam Factory ${ }^{18}$. No events were observed for ${ }^{32,33} \mathrm{~F},{ }^{35,36} \mathrm{Ne}$, and ${ }^{38} \mathrm{Na}$ and only one event for ${ }^{39} \mathrm{Na}$ after extensive investigation. Ref. 18 suggests that ${ }^{31} \mathrm{~F}$ and ${ }^{34} \mathrm{Ne}$ are the heaviest bound isotopes of fluorine and neon, respectively. The calculations summarized in this paper suggest that sodium nuclei more massive than ${ }^{38} \mathrm{Na}$ could exist.

An et al. ${ }^{19}$ perform a theoretical study of $Z=8-12$ isotopes in the relativistic mean field model. Ref. 19 notes that the last bound neutron-rich nuclei with $Z=8,9,10,11$, and 12 varies with the theoretical models with upper limit mass values of $28,33,43,45$, and 46 , respectively. The sodium A = 45 mass limit is consistent with the predictions of this paper that also predicts ${ }^{45} \mathrm{Na}$ as the limiting neutron excess sodium system.

\subsection{Calculational Methodology}

A variety of models could be applied to the investigation of neutron excess nuclei. These vary in sophistication, but the proposed model utilizes a basic single-particle approach. This is a reasonable first step because there are uncertainties in the nuclear potential that likely are more significant than the limitations introduced by a single-particle approach.

Since the method for calculating single-particle energies in a spherically symmetric potential is wellestablished only salient features are provided. The model used to describe the particle plus core system represents an application of the standard method of Lukasiak and Sobiczewski ${ }^{20}$ and Petrovich et. al. ${ }^{21}$

The binding energy $E_{N L S}$ of a particle in the field of a nuclear core is obtained by solving the radial Schrödinger Equation

$$
\left[\frac{\hbar^{2}}{2 \mu}\left(\frac{d^{2}}{d r^{2}}-\frac{L(L+1)}{r^{2}}\right)-E_{N L S J}-V_{L S J}(r)\right] U_{N L S J}(r)=0(1)
$$

where $r$ is the radial coordinate defining the relative motion of the nuclear core and the particle; $V_{L S J}(r)$ is the model interaction; $E_{N L S J}$ is the core plus particle binding energy; $U_{N L S J}(r)$ is the radial wave function; and $\mathrm{L}, \mathrm{S}$, and $\mathrm{J}$ are the orbital, spin, and total angular momentum quantum numbers, respectively. The $\mathrm{N}$ quantum number is the radial quantum number, and $\mu$ is the reduced mass.

The method of searching for $E_{\text {NLS }}$ is provided by Brown, Gunn, and Gould 22 , and the methodology of Ref. 23 is utilized to obtain a converged solution. Refs. 8 - 12 and 21 provide a more complete description of the model, its numerical solution, and further definition of the individual terms appearing in Eq. 1.

\subsection{Nuclear Interaction}

Nuclear stability with respect to alpha decay, beta decay, positron decay, and electron capture is addressed using the method previously published by the author and coworkers ${ }^{8-12,} 21$ that is similar to the approach of Ref. 24. The single-particle level spectrum is generated using a Woods-Saxon potential. Parameters of the potential are obtained from a fit to the particle levels in ${ }^{209} \mathrm{~Pb}$ and ${ }^{209} \mathrm{Bi}$ performed by 
Rost $^{25}$. The central potential strength of the Rost interaction ${ }^{25}$ has a standard form and can be explicitly defined as

$$
V_{0}=51.6\left[1 \pm 0.73^{\frac{N-Z}{A}}\right]_{(2)}
$$

where the upper (lower) sign applies to protons (neutrons). The remaining parameters were held constant and are given by Rost ${ }^{25}: r_{0}=1.262(1.295) \mathrm{fm}, r_{\text {so }}=0.908(1.194) \mathrm{fm}, \mathrm{a}=0.70(0.70) \mathrm{fm}$, and $\mathrm{y}=17.5$ (28.2) for protons (neutrons) ${ }^{21,25}$. The spin-orbit interaction strength $V_{\text {so }}$ is related to $y$ by the relationship ${ }^{25}$ :

$$
V_{\text {so }}=\frac{y V_{0}}{180}
$$

The scaling relationships of Eqs. 2 and 3 yield reasonable fits to observed single-particles levels in ${ }^{120} \mathrm{Sn}$ and ${ }^{138} \mathrm{Ba}$. The pairing correction term of Blomqvist and Wahlborn ${ }^{26}$ is used in the calculations presented herein. The pairing correction improves the predicted energies of occupied levels in ${ }^{120} \mathrm{Sn},{ }^{138} \mathrm{Ba}$, and ${ }^{208} \mathrm{~Pb}^{21}$.

When applied to specific nuclei, this methodology requires modification. For example, Ray and Hodgson ${ }^{27}$ note that ${ }^{40} \mathrm{Ca}$ and ${ }^{48} \mathrm{Ca}$ require different potentials to properly fit their single-particle level structure. Schwierz, Wiedenhöver, and Volya ${ }^{28}$ also investigated ${ }^{40} \mathrm{Ca}$ and ${ }^{48} \mathrm{Ca}$ and noted that a proper fit to the single-particle levels required a different potential for each energy level. Difficulties in the selection of an appropriate potential is an additional motivation for the utilization of a single-particle model and was noted in studies of neutron excess calcium ${ }^{8}$, iron ${ }^{9}$, fluorine ${ }^{10}$, zinc ${ }^{11}$, and neon ${ }^{12}$ nuclei. Similar issues also apply to sodium systems.

In view of the results of Refs. 27 and 28, the following modification is made to obtain the sodium potential strength $\left(\mathrm{V}_{\mathrm{A}}\right)$ :

$$
V_{A}=51.6 \lambda\left[1 \pm 0.73^{\frac{N-Z}{A}}\right][1 \pm a(A)] \operatorname{MeV}(4)
$$

where $\lambda$ is a potential strength multiplier that is selected to ensure consistency with available data, and $a(A)$ is a constant that is introduced to account for the variations in potential strength with $A^{27,28}$. In previous excess neutron nuclei calculations for calcium ${ }^{8}$, iron ${ }^{9}$, and zinc ${ }^{11}$, a value of $\lambda=1.0$ was utilized. $A \lambda$ value of 1.5 for fluorine ${ }^{10}$ and neon ${ }^{12}$ were determined by the available experimental data ${ }^{29-31}$. Given the proximity of fluorine and neon, a value of $\lambda=1.5$ is also utilized for sodium. Since the paper's primary purpose is investigation of the neutron excess nuclei, determining a common a(A) value for the heaviest sodium systems is desirable.

The heaviest mass A $=11$ isotope ${ }^{29-31}$ suggested experimentally is ${ }^{35} \mathrm{Na}$. Given the expected order of energy levels, ${ }^{35} \mathrm{Na}$ would have a $1 f_{7 / 2}$ neutron single-particle level structure. Isotopes heavier than ${ }^{35} \mathrm{Ne}$ 
would require filling of the $1 f_{7 / 2}$ and the more weakly bound $2 p_{3 / 2}$ and $2 p_{1 / 2}$ neutron single-particle levels. The possibility of bound sodium isotopes with $A \geq 35$ is addressed in subsequent discussion.

Calculations incorporated into the Japanese nuclear data compilation ${ }^{31}$ provide a calculated half-life for ${ }^{37} \mathrm{Na}$. Ref. 19 suggests that the last bound neutron-rich sodium nucleus has a value of $A=45$.

\subsection{Calculation of Half-Lives}

Using Eq. 4, single-particle levels are calculated for $A \geq 20$ sodium isotopes. $A \geq 20$ sodium nuclei were evaluated for stability with respect to alpha decay, beta decay, positron decay, and electron capture. These calculations were performed to ensure that the nuclear structure contained no interloping states or structural defects, and that any decay modes in conflict with data were identified.

The decay modes and half-lives of $45 \geq A \geq 20$ sodium isotopes are summarized in Table I, and compared to available data ${ }^{29-31}$ and calculations incorporated in the Japanese data compilation ${ }^{31}$. The alpha decay energies are calculated using the relationship based on Ref. 32

$$
Q_{\alpha}=28.3 \mathrm{MeV}-2 S_{n}-2 S_{p}(5)
$$

where $S_{n}$ and $S_{p}$ are the binding energies of the last occupied neutron and proton single-particle levels, respectively. Alpha decay half-lives can be estimated from $Q_{\alpha}$ using standard relationships ${ }^{20}$. Fortunately, no alpha decay modes occurred in the Table I summary of $45 \geq A \geq 20$ sodium isotope decay properties.

The beta decay half-lives are determined following the log ft methodology of Wong ${ }^{32}$. Allowed (first forbidden) transition half-lives were derived using the values of $\log \mathrm{ft}=5$ (8). Given the uncertainties in the calculated level energies, second and higher order forbidden transitions were not determined. Positron and electron capture half-lives were determined following the approach of Ref. 20.

\subsection{Model Issues}

Spherical single-particle energy level calculations produce reasonable results for alpha, beta, positron, and electron capture transitions ${ }^{8-12,24-28}$. However, these calculations are not expected to accurately model the very short-lived proton decay mode of ${ }^{18} \mathrm{Na}$ and ${ }^{19} \mathrm{Na}^{29-31}$. Since ${ }^{18} \mathrm{Na}$ and ${ }^{19} \mathrm{Na}$ are far removed from the neutron excess sodium isotopes of interest in this paper, they are not addressed. In addition, very heavy sodium isotopes have the potential to decay via neutron emission modes. However, these decays have not been observed in sodium ${ }^{29-31}$. The single-particle model is not the best approach for neutron emission calculations, and these decay modes are not included in this paper. Therefore, the results for the heaviest neutron excess sodium nuclei only include the alpha decay, beta decay, positron decay, and electron capture modes.

Except as noted previously, the single-particle model should provide reasonable results for the systems considered in the paper. Since the focus of this paper is the more massive sodium nuclei, single-particle methods provide the desired results for the most important nuclei considered in this study, and their decay via the alpha decay, beta decay, positron decay, and electron capture pathways.

\subsection{Results and Discussion}


Using Eq. 4, the a(A) value was varied in increments of $0.001-0.005$ to assess the applicability of the proposed model to predict the decay properties of most $45 \geq A \geq 20$ sodium isotopes. In view of uncertainties in the model and associated interaction, a smaller increment was not deemed to be justified for most sodium systems. However, for nuclei that have half-lives that deviate from stability trends in neighboring systems, a smaller increment was utilized. For example, a(A) was adjusted in increments of 0.00001 for the stable ${ }^{23} \mathrm{Na}$ system.

The issues associated with fitting all calcium, iron, fluorine, zinc, and neon nuclei with a single potential 27,28 were noted in Refs. 8-12. These considerations are also applicable to the sodium systems considered in this paper.

Table I summarizes the complete set of $45 \geq A \geq 20$ sodium isotopes considered in this paper. The lighter $45 \geq A \geq 20$ sodium isotopes fill the $1 d_{5 / 2}\left({ }^{20} \mathrm{Na}-{ }^{25} \mathrm{Na}\right), 2 \mathrm{~s}_{1 / 2}\left({ }^{26} \mathrm{Na}\right.$ and $\left.{ }^{27} \mathrm{Na}\right), 1 d_{3 / 2}\left({ }^{28} \mathrm{Na}-{ }^{31} \mathrm{Na}\right)$, and $1 f_{7 / 2}\left({ }^{32} \mathrm{Na}-{ }^{35} \mathrm{Na}\right)$ neutron single-particle levels. These systems are the heaviest sodium systems noted in Ref. 29 - 31 that have been observed experimentally. ${ }^{32} \mathrm{Na}-{ }^{35} \mathrm{Na}$ partially fill the $1 f_{7 / 2}$ neutron single-particle shell. Given the extrapolation used in formulating the single-particle potential of Eq. 4, the results become more uncertain due to the paucity of data for $A>35$ sodium isotopes. The heavier $45 \geq A \geq$ 20 sodium isotopes that complete the $1 f_{7 / 2}$, and fill the $2 p_{3 / 2}$, and $2 p_{1 / 2}$ neutron single-particle levels are also summarized in Table I. These systems represent the heaviest possible neutron excess systems that would occur in the $Z=11$ system. 
Table I

Calculated Single-Particle and Experimental Decay Properties of Sodium Systems with $20 \leq \mathrm{A} \leq$ 45

\begin{tabular}{l|l|l|l}
\hline Nuclide & a(A) & Half-Life $(\text { Decay Mode })^{\mathrm{a}, \mathrm{b}}$ & \\
\hline${ }^{20} \mathrm{Na}$ & +0.013 & $448 \mathrm{~ms}\left(\beta^{+}\right)^{\mathrm{a}}$ & This Work \\
\hline${ }^{21} \mathrm{Na}$ & +0.054 & $22.48 \mathrm{~s}\left(\beta^{+}\right)^{\mathrm{a}}$ & $443 \mathrm{~ms}\left(\beta^{+}\right)^{\mathrm{c}}$ \\
${ }^{22} \mathrm{Na}$ & +0.05949 & $2.604 \mathrm{yr}$ & $22.6 \mathrm{~s}\left(\beta^{+}\right)^{\mathrm{c}}$ \\
${ }^{23} \mathrm{Na}$ & +0.02558 & Stable & $2.57 \mathrm{yr}\left(\beta^{+}\right)^{\mathrm{c}}$ \\
\hline${ }^{24} \mathrm{Na}$ & -0.00063 & $14.97 \mathrm{~h}\left(\beta^{-}\right)^{\mathrm{a}}$ & Stable \\
\hline${ }^{25} \mathrm{Na}$ & -0.0036 & $59.3 \mathrm{~s}\left(\beta^{-}\right)^{\mathrm{a}}$ & $15.0 \mathrm{~h}\left(\beta^{-}\right)^{\mathrm{d}}$ \\
\hline${ }^{26} \mathrm{Na}$ & +0.025 & $1.0711 \mathrm{~s}\left(\beta^{-}\right)^{\mathrm{a}}$ & $59.4 \mathrm{~s}\left(\beta^{-}\right)^{\mathrm{d}}$ \\
${ }^{27} \mathrm{Na}$ & +0.033 & $290 \mathrm{~ms}\left(\beta^{-}\right)^{\mathrm{a}}$ & $1.08 \mathrm{~s}\left(\beta^{-}\right)^{\mathrm{d}}$ \\
\hline${ }^{28} \mathrm{Na}$ & +0.049 & $30.5 \mathrm{~ms}\left(\beta^{-}\right)^{\mathrm{a}}$ & $294 \mathrm{~ms}\left(\beta^{-}\right)^{\mathrm{d}}$ \\
\hline${ }^{29} \mathrm{Na}$ & +0.0093 & $44 \mathrm{~ms}\left(\beta^{-}\right)^{\mathrm{a}}$ & $30.6 \mathrm{~ms}\left(\beta^{-}\right)^{\mathrm{e}}$ \\
\hline${ }^{30} \mathrm{Na}$ & -0.016 & $50 \mathrm{~ms}\left(\beta^{-}\right)^{\mathrm{a}}$ & $44.0 \mathrm{~ms}\left(\beta^{-}\right)^{\mathrm{e}}$ \\
\hline${ }^{31} \mathrm{Na}$ & +0.0226 & $17.2 \mathrm{~ms}\left(\beta^{-}\right)^{\mathrm{a}}$ & $49.8 \mathrm{~ms}\left(\beta^{-}\right)^{\mathrm{e}}$ \\
\hline${ }^{32} \mathrm{Na}$ & +0.022 & $13.2 \mathrm{~ms}\left(\beta^{-}\right)^{\mathrm{a}}$ & $17.2 \mathrm{~ms}\left(\beta^{-}\right)^{\mathrm{e}}$ \\
\hline${ }^{33} \mathrm{Na}$ & +0.037 & $8.3 \mathrm{~ms}\left(\beta^{-}\right)^{\mathrm{a}}$ & $13.2 \mathrm{~ms}\left(\beta^{-}\right)^{\mathrm{e}}$ \\
\hline${ }^{34} \mathrm{Na}$ & +0.060 & $5.0 \mathrm{~ms}\left(\beta^{-}\right)^{\mathrm{a}}$ & $8.30 \mathrm{~ms}\left(\beta^{-}\right)^{\mathrm{e}}$ \\
\hline${ }^{35} \mathrm{Na}$ & +0.160 & $1.5 \mathrm{~ms}\left(\beta^{-}\right)^{\mathrm{a}}$ & $5.02 \mathrm{~ms}\left(\beta^{-}\right)^{\mathrm{e}}$ \\
\hline
\end{tabular}




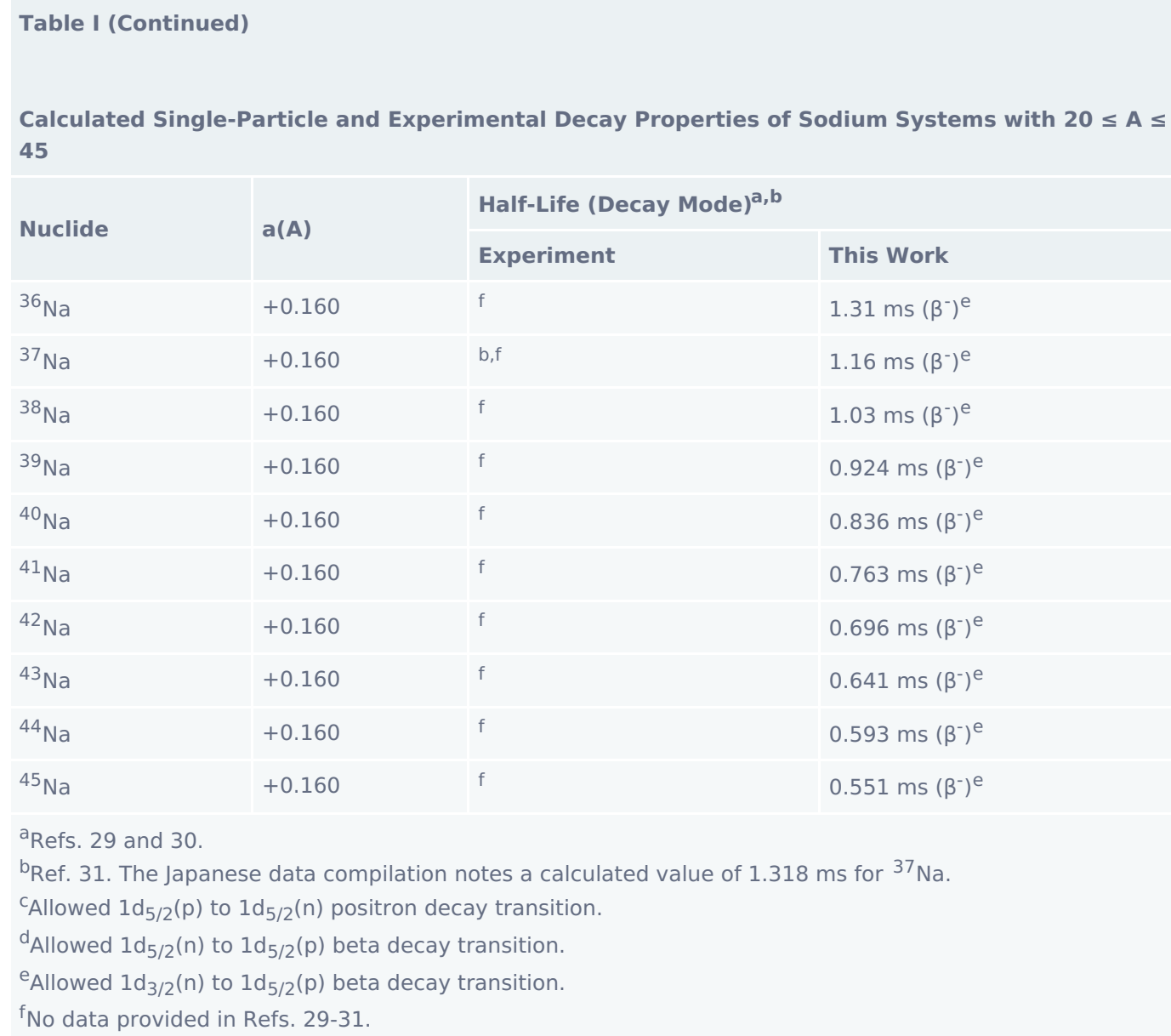

The neutron excess systems summarized in Table I were based on an evaluation of alpha, beta, electron capture, and positron decay modes. Other decay modes that could possibly occur in neutron excess systems (e.g., $n$ and $2 n$ ) are not readily evaluated using a single particle model, and were not evaluated. The results of Table I must be viewed with this limitation. However, since the neutron and proton decay modes tend to be much shorter than the alpha, beta, electron capture, and positron decay modes ${ }^{29-31}$, the model results provide upper bounds on the half-lives of neutron excess sodium isotopes.

\section{$6.135 \geq A \geq 20$ Sodium Isotopes with Experimental Half-Life Data}

${ }^{20} \mathrm{Na}$ to ${ }^{25} \mathrm{Na}$ systems were best fit with a(A) values between -0.0036 and 0.05949 with an average value of about 0.025 . The ${ }^{20} \mathrm{Na}$ to ${ }^{25} \mathrm{Na}$ nuclei fill the $1 d_{5 / 2}$ neutron shell. ${ }^{26} \mathrm{Na}$ and ${ }^{27} \mathrm{Na}$ fill the $2 \mathrm{~s}_{1 / 2}$ neutron shell and are best fit with $\mathrm{a}(\mathrm{A})$ values of 0.025 and 0.033 , respectively with an average value of 0.029 .

${ }^{28} \mathrm{Na}$ to ${ }^{31} \mathrm{Na}$ systems were best fit with $\mathrm{a}(\mathrm{A})$ values between -0.016 and 0.049 with an average value of about 0.016 . The ${ }^{28} \mathrm{Na}$ to ${ }^{31} \mathrm{Na}$ nuclei fill the $1 d_{3 / 2}$ neutron shell.

The heaviest known sodium neutron excess systems (i.e., ${ }^{32} \mathrm{Na}-{ }^{35} \mathrm{Na}$ ) partially fill the $1 f_{7 / 2}$ neutron shell. There is no experimental half-life data for $A$ > 35 sodium systems.

The ${ }^{32} \mathrm{Na}-{ }^{35} \mathrm{Na}$ systems were best fit with a(A) values between 0.022 and 0.160 , with an average value of about 0.070 . The 0.160 value for the heaviest known sodium system $\left({ }^{35} \mathrm{Na}\right)$ is larger than the limiting 
values noted for calcium $(0.090)^{8}$, iron $(0.115)^{9}$, fluorine $(0.115)^{10}$, zinc $(0.095)^{11}$, and neon $(0.119)^{12}$. Since it is the heaviest experimentally observed sodium system, the ${ }^{35} \mathrm{Na}$ value is used to extrapolate the half-lives of ${ }^{36} \mathrm{Na}$ and heavier sodium nuclei. The selected value of 0.160 is similar to the 0.119 value utilized in the neighboring neon system ${ }^{12}$.

Table I lists the half-life of the limiting decay transition (i.e., the transition that has the shortest decay half-life). For example, ${ }^{26} \mathrm{Na}$ has four beta decay transitions that are possible within the scope of the aforementioned single-particle model (i.e., allowed $1 d_{5 / 2}(n)$ to $1 d_{5 / 2}(p)$ [1.08 s], allowed $1 d_{5 / 2}(n)$ to $1 d_{3 / 2}(p)$ [1.58 min], allowed $2 s_{1 / 2}(n)$ to $2 s_{1 / 2}(p)$ [1.36 s], and first forbidden $1 p_{1 / 2}(n)$ to $1 d_{5 / 2}(p)$ [257 d]. For ${ }^{26} \mathrm{Na}$, the limiting beta decay mode is the allowed $1 d_{5 / 2}(n)$ to $1 d_{5 / 2}(p)[1.08 \mathrm{~s}]$ transition.

As noted in Table I, the model predicts the proper decay mode for the known $35 \geq A \geq 20$ sodium nuclei29-31. The results for the known systems summarized in Table I suggest that the model predictions of the neutron excess sodium systems are reasonably credible.

For nuclei filling the $1 d_{5 / 2}$ neutron shell, model predictions for ${ }^{20} \mathrm{Na},{ }^{21} \mathrm{Na},{ }^{22} \mathrm{Na},{ }^{24} \mathrm{Na}$, and ${ }^{25} \mathrm{Na}$ are within about $1.5 \%$ of the experimental positron or beta decay half-lives ${ }^{29} .{ }^{23} \mathrm{Na}$ is correctly determined to be a stable system. ${ }^{20} \mathrm{Na},{ }^{21} \mathrm{Na}$, and ${ }^{22} \mathrm{Na}$ decays by an allowed $1 d_{5 / 2}(p)$ to $1 d_{5 / 2}(n)$ positron decay transition. The ${ }^{24} \mathrm{Na}$, and ${ }^{25} \mathrm{Na}$ systems decay by an allowed $1 d_{5 / 2}(n)$ to $1 d_{5 / 2}(p)$ beta decay transition.

The $2 \mathrm{~s}_{1 / 2}$ systems, ${ }^{26} \mathrm{Na}$ and ${ }^{27} \mathrm{Na}$, are within $1.5 \%$ of their respective experimental beta decay halflives ${ }^{29}$. Both ${ }^{26} \mathrm{Na}$ and ${ }^{27} \mathrm{Na}$ decay by an allowed $1 d_{5 / 2}(\mathrm{n})$ to $1 d_{5 / 2}(p)$ beta decay transition.

${ }^{28} \mathrm{Na},{ }^{29} \mathrm{Na},{ }^{30} \mathrm{Na}$, and ${ }^{31} \mathrm{Na}$ fill the $1 \mathrm{~d}_{3 / 2}$ neutron shell. The ${ }^{28} \mathrm{Na},{ }^{29} \mathrm{Na},{ }^{30} \mathrm{Na}$, and ${ }^{31} \mathrm{Na}$ systems decay by an allowed $1 d_{3 / 2}(n)$ to $1 d_{5 / 2}(p)$ beta decay transition, and their beta decay half-lives are within $0.5 \%$ of the measured values ${ }^{29}$.

The $1 f_{7 / 2}$ systems, ${ }^{32} \mathrm{Na},{ }^{33} \mathrm{Na},{ }^{34} \mathrm{Na}$, and ${ }^{35} \mathrm{Na}$, are within $0.5 \%$ of their respective experimental beta decay half-lives ${ }^{29}$. These systems decay by an allowed $1 d_{3 / 2}(n)$ to $1 d_{5 / 2}(p)$ beta decay transition. These are the heaviest sodium nuclides that have measured decay half-life values and beta decay transition information ${ }^{29}$.

\section{$6.245 \geq A \geq 36$ Sodium Isotopes}

As noted in the previous section, the limiting a(A) value of 0.160 was derived from the heaviest sodium isotope $\left({ }^{35} \mathrm{Na}\right)$ measured experimentally ${ }^{29}$. This a(A) value was used for all $45 \geq A \geq 36$ sodium systems.

Table I also summarizes calculated single-particle decay properties of sodium systems with $45 \geq A \geq$ 36. Although experimental data for $45 \geq A \geq 36$ sodium systems are not available ${ }^{29-31}$, these are nuclei of interest in astrophysical applications ${ }^{1-7,}$ 13-17.

The existence of $45 \geq A \geq 36$ sodium systems as predicted by the proposed model is dependent on the characteristics of the interaction of Eq. 4. Although the existence of some of these systems may be an artifact of the model interaction, their study is of critical importance in understanding the role of neutron excess sodium systems in nucleosynthesis.

The ${ }^{36} \mathrm{Na}-{ }^{39} \mathrm{Na}$ systems complete filling the $1 \mathrm{f}_{7 / 2}$ neutron single-particle energy level. These systems 
have beta decay half-life values that decrease from 1.31 to $0.924 \mathrm{~ms}$, respectively. Although no data is available for the ${ }^{36} \mathrm{Na}-{ }^{39} \mathrm{Na}$ systems, the calculated beta decay half-life for ${ }^{37} \mathrm{Ne}$ of $1.16 \mathrm{~ms}$ is consistent with the calculations of Ref. 31 . The ${ }^{36} \mathrm{Na}-{ }^{39} \mathrm{Na}$ systems decay through an allowed $1 d_{3 / 2}(n)$ to $1 d_{5 / 2}(p)$ beta decay transition.

The ${ }^{40} \mathrm{Na}-{ }^{43} \mathrm{Na}$ systems fill the $2 p_{3 / 2}$ neutron shell. These systems also decay through an allowed $1 d_{3 / 2}(n)$ to $1 d_{5 / 2}(p)$ beta decay transition. The ${ }^{40} \mathrm{Na}-{ }^{43} \mathrm{Na}$ beta decay half-lives decrease from 0.836 to $0.641 \mathrm{~ms}$, respectively.

The ${ }^{44} \mathrm{Na}$ and ${ }^{45} \mathrm{Na}$ systems fill the $2 \mathrm{p}_{1 / 2}$ neutron shell. In a similar manner, these systems decay through an allowed $1 d_{3 / 2}(n)$ to $1 d_{5 / 2}(p)$ beta decay transition. The ${ }^{44} \mathrm{Na}$ and ${ }^{45} \mathrm{Na}$ half-lives are 0.593 and $0.551 \mathrm{~ms}$, respectively.

No sodium isotopes with $A>45$ are predicted by the model. This occurs because the $2 \mathrm{p}_{1 / 2}$ neutron single-particle level is the last bound neutron state, and only 34 neutrons are bound in sodium systems. However, in view of the model potential uncertainties, the calculated properties of the heaviest sodium systems summarized in Table I are not definitive.

The predicted $A=36-45$ sodium isotopes have no experimental half-life data, but the model predicts beta decay half-lives in the range of $0.551-1.31 \mathrm{~ms}$. Based on calculations in $Z=9,10,20,26$, and 30 systems ${ }^{8-12}$, these results likely overestimate the beta decay half-lives of these neutron excess sodium nuclei. The model results are also likely to be an overestimate of the half-lives because the single-particle level calculations do not evaluate the short-lived neutron decay modes in the A = $36-45$ sodium nuclei.

\subsection{Conclusions}

Single-particle level calculations suggest that neutron excess sodium isotopes terminate with ${ }^{45} \mathrm{Na}$ and filling of the $2 p_{1 / 2}$ neutron single-particle level. The $36 \leq A \leq 45$

sodium systems have predicted beta decay half-lives in the 0.551 - $1.31 \mathrm{~ms}$ range, and likely overestimate the actual half-life values.

\section{References}

1) D. M. Siegel and B. D. Metzger, Phys. Rev. Lett. 119, 231102 (2017).

2) National Academy of Sciences Report No. 11796, Scientific Opportunities with a Rare-Isotope Facility in the United States, Washington DC: National Research Council (2007).

3) N. Fukuda et al., J. Phys. Soc. Jpn. 87, 014202 (2018).

4) Y. Shimizu et al., J. Phys. Soc. Jpn. 87, 014203 (2018).

5) J. Kurcewicz et al., Phys. Lett. B 717, 371 (2012).

6) T. Baumann et al., Nature 449, 1022 (2007).

7) O. B. Tarasov et al., Phys. Rev. C 87, 054612 (2013).

8) J. J. Bevelacqua, Decay Characteristics of Neutron Excess Calcium Nuclei, Physics Essays 31 (4), 462 (2018).

9) J. J. Bevelacqua, Decay Characteristics of Neutron Excess Iron Nuclei, Physics Essays 32 (2), 175 (2020). 
10) J. J. Bevelacqua, Decay Characteristics of Neutron Excess Fluorine Nuclei, QEIOS

24XLL9, 1 (2020). https://doi.org/10.32388/24XLL9.

11) J. J. Bevelacqua, Decay Characteristics of Neutron Excess Zinc Nuclei, QEIOS, JZI1LG, 1 (2020). https://doi.org/10.32388/JZI1LG.

12) J. J. Bevelacqua, Decay Characteristics of Neutron Excess Neon Nuclei, QEIOS

1WR291, 1 (2021). https://doi.org/10.32388/1WR291.

13) M. Terasawa, K. Sumiyosh, T. Kajino, G. J. Mathews, and I. Tanihata, New Nuclear Reaction Flow during r-Process Nucleosynthesis in Supernovae: Critical Role of Light Neutron-Rich Nuclei,

https://cds.cern.ch/record/509832/files/0107368.pdf.

14) A. Recio-Blanco, P. de Laverny, C. Worley, N. C. Santos, C. Melo, and G. Israelian, Astron. Astrophys 538, A117 (2012).

15) N. Mowlavi, A. Jorissen, and M. Arnould, Astron. Astrophys. 334, 153 (1998).

16) O. S. Kirsebom et al., Phys. Rev. C 100, 065805 (2019).

17) O. S. Kirsebom et al., Phys. Rev. Lett. 123, 262701 (2019).

18) D. S. Ahn, N. Fukuda, H. Geissel, N. Inabe, N. Iwasa, T. Kubo, K. Kusaka, D. J. Morrissey, D. Murai, and T. Nakamura, et al. Physical Review Letters 123, 212501 (2019).

19) R. An, G.-F. Shen, S.-S. Zhang, and L.-S. Geng, Chinese Physics C 44, No. 7, 074101 (2020).

20) A. Lukasiak and A. Sobiczewski, Acta Phys. Pol. B6, 147 (1975).

21) F. Petrovich, R. J. Philpott, D. Robson, J. J. Bevelacqua, M. Golin, and D. Stanley, Phys. Rev. Lett. 37, 558 (1976).

22) G. E. Brown, J. H. Gunn, and P. Gould, Nucl. Phys. 46, 598 (1963).

23) L. Fox and E. T. Godwin, Proc. Cambridge Philos. Soc. 45, 373(1949).

24) S. Hofmann and G. Münzenberg, Rev. Mod. Phys. 72, 733 (2000).

25) E. Rost, Phys. Lett. 26B, 184 (1968).

26) J. Blomqvist and S. Wahlborn, Ark. Fys. 16, 545 (1959).

27) L. Ray and P. E. Hodgson, Phys. Rev. C 20, 2403 (1979).

28) N.Schwierz, I. Wiedenhöver, and A. Volya, arXiv:0709.3525v1 [nucl-th] 21 Sep 2007.

29) E. M. Baum, M. C. Ernesti, H. D. Knox, T. R. Miller, and A. M. Watson, Nuclides and Isotopes - Chart of the Nuclides, 17th ed, Knolls Atomic Power Laboratory (2010).

30) National Nuclear Data Center, Brookhaven National Laboratory. NuDat (Nuclear Structure and Decay Data). http://www.nndc.bnl.gov/nudat2/ (accessed 08 June, 2020).

31) H. Koura et al., Chart of the Nuclides 2018, Japanese Nuclear Data Committee and Nuclear Data Center, Japanese Atomic Energy Agency (2018).

32) C. Y. Wong, Phys. Lett. 21, 688 (1966). 
